\title{
On a 2+1-Dimensional Whitham-Broer-Kaup System: A Resonant NLS Connection
}

\author{
By Colin Rogers and Oktay Pashaev
}

It is established that the Whitham-Broer-Kaup shallow water system and the "resonant" nonlinear Schrödinger equation are equivalent. A symmetric integrable 2+1-dimensional version of the Whitham-Broer-Kaup system is constructed which, in turn, is equivalent to a recently introduced resonant Davey-Stewartson I system incorporating a Madelung-Bohm type quantum potential. A bilinear representation is adopted and resonant solitonic interaction in this new 2+1-dimensional Kaup-Broer system is exhibited.

\section{Introduction}

In [1], Kaup derived via a higher-order Boussinesq-type approximation the nonlinear system

$$
\begin{aligned}
\Pi_{T} & =\Phi_{X X}+\delta^{2}\left(\frac{1}{3}-\sigma\right) \Phi_{X X X X}-\epsilon\left(\Phi_{X} \Pi\right)_{X}, \\
\Pi & =\Phi_{T}+\frac{1}{2} \epsilon \Phi_{x}^{2}
\end{aligned}
$$

descriptive of water wave propagation in a long narrow channel. Therein, $\delta$ is a depth/wave length parameter, $\epsilon$ is a wave amplitude/depth ratio, while $\Phi$ is the velocity potential and $\Pi$ is the amplitude of the surface wave. The system had been previously obtained in [2] in a study of eigenvalue problems associated with the inverse scattering method. The detailed IST analysis was

\footnotetext{
Address for correspondence: Colin Rogers, Department of Applied Mathematics, Australian Research Council Centre of Excellence for Mathematics \& Statistics of Complex Systems. e-mail: c.rogers@unsw.edu.au 
carried out in [1]. A system equivalent to (1) was obtained independently by Broer $[3,4]$ in a Hamiltonian treatment of long wave propagation.

On introduction of the change of variables

$$
\begin{aligned}
x & =\alpha X, t=\beta T, \\
\Phi_{x} & =\frac{2 \delta}{\epsilon} \sqrt{\left(\frac{1}{3}-\sigma\right)} u, \\
\Pi & =-\frac{2 \delta^{2}}{\epsilon}\left(\frac{1}{3}-\sigma\right) \alpha^{2}\left(h-u_{x}-\frac{\alpha^{2}}{2 \beta^{2}}\right)
\end{aligned}
$$

where

$$
\beta=-\alpha^{2} \delta \sqrt{\frac{1}{3}-\sigma},
$$

one obtains what is sometimes termed the Broer-Kaup system [5]

$$
\begin{aligned}
& h_{t}-h_{x x}+2(u h)_{x}=0, \\
& u_{t}-h_{x}+u_{x x}+2 u u_{x}=0 .
\end{aligned}
$$

In fact, the provenance of both of the systems (1) and (3) goes back to a paper by Whitham [6] in 1967 on a variational approach to nonlinear wave theory. Therein, in a dispersive correction to irrotational shallow water theory, the long wave system

$$
\begin{aligned}
& h_{t}+\left(h F_{x_{i}}\right)_{x_{i}}+\frac{1}{3} h_{0}^{2} \nabla^{4} F=0, \\
& F_{t}+\frac{1}{2} F_{x_{i}}^{2}+g h=0,
\end{aligned}
$$

was derived. Here, $F$ represents the velocity potential at the water surface $y=h(\mathbf{x}, t)$ while the approximation assumes that the amplitude parameter $a / h_{0}$ is small in addition to $h_{0}^{2} / \lambda^{2}$ where $\lambda$ is a typical wave length. In the $1+1$-dimensional reduction, the Whitham system (4) and the system (1) are equivalent, up to scaling, under the transformation

$$
\begin{aligned}
& \Pi=\frac{(1-g h)}{\epsilon}, \Phi=\frac{(F+T)}{\epsilon}, \\
& t=T .
\end{aligned}
$$

The integrability of the Broer-Kaup system as validated by the existence of its inverse scattering scheme [1], and its invariance under a Bäcklund transformation [5], implies that the equivalent $1+1$-dimensional Whitham system is integrable. However, the $n+1$-dimensional Whitham system (4) appears not to be integrable for $n>1$ and so does not provide an integrable higher-dimensional extension of the Broer-Kaup system. However, it will be shown here that an integrable symmetric generalization may be obtained via a $2+1$-dimensional resonant Davey-Stewartson I system recently introduced 
by Rogers et al. [7] in the context of a capillarity model. A recent Painlevé analysis of this system is consistent with its integrability [8].

A nonlinear Schrödinger (NLS) equation of the type

$$
i \Psi_{t}+\nabla^{2} \Psi+\nu|\Psi|^{2} \Psi=s\left(\frac{\nabla^{2}|\Psi|}{|\Psi|}\right) \Psi
$$

incorporating a de Broglie-Bohm type quantum potential term [9, 10], namely $\nabla^{2}|\Psi| /|\Psi|$, could be derived via Maxwell's equations in the setting of the self-trapping of optical beams by Wagner et al. in [11]. In that context, $s<1$ and reduction of (6) may be readily made to the conventional NLS equation with the de Broglie-Bohm term removed [12].

A $1+1$-dimensional variant (6) of the cubic NLS equation was subsequently derived by Pashaev and Lee [13] in the setting of the Jackiw-Teitelbaum gravity model in general relativity. However, there the parameter $s>1$ and reduction of (6) to the conventional NLS equation are no longer available. In that case, rather reduction may be made to a coupled nonlinear system of the type [13]

$$
\begin{aligned}
& \hbar \partial e^{+} / \partial t+\left(\hbar^{2} / 2\right) \nabla^{2} e^{+}-v e^{+} e^{-} e^{+}=0, \\
& \quad-\hbar \partial e^{-} / \partial t+\left(\hbar^{2} / 2\right) \nabla^{2} e^{-}-v e^{+} e^{-} e^{-}=0 .
\end{aligned}
$$

The appearance of resonant solitonic behavior in the latter system involving novel fusion and fission solitonic phenomena has led to introduction of the terminology resonant NLS equation for (6) in the case $s>1$.

The resonant NLS equation has subsequently been derived in plasma physics, where it describes the propagation of 1-dimensional, long magneto-acoustic waves in a cold collisionless plasma subject to a transverse magnetic field [14]. An auto-Bäcklund transformation was constructed therein. The resonant NLS equation as an integrable capillarity model was introduced in [15]. Indeed, it turns out that a wide class of NLS equations with underlying Hamiltonian structure may be reduced to consideration of the resonant NLS equation [16].

Here, it is shown that, remarkably, the well-known Whitham-Broer-Kaup system of shallow water theory and the $1+1$-dimensional resonant NLS equation are equivalent. Further, an integrable 2+1-dimensional symmetric version of the Whitham-Broer-Kaup system is constructed and a bilinear representation is used to isolate solitonic solutions which exhibit resonance.

\section{A 2+1-dimensional Whitham-Broer-Kaup system}

The 2+1-dimensional resonant Davey-Stewartson system as introduced in Rogers et al. in [7] adopts the form

$$
\begin{gathered}
i \Psi_{t}+\nabla^{2} \Psi+(\delta-1)\left(\frac{\nabla^{2}|\Psi|}{|\Psi|}\right)^{2} \Psi+\gamma|\Psi|^{2} \Psi+\frac{1}{2} \Pi \Psi=0, \\
\Pi_{x x}-\Pi_{y y}+4 \gamma\left(|\Psi|^{2}\right)_{x x}=0 .
\end{gathered}
$$


The decomposition $\Psi=e^{R-i S}$ results in the system

$$
\begin{aligned}
& R_{t}-S_{x x}-S_{y y}-2 R_{x} S_{x}-2 R_{y} S_{y}=0, \\
& S_{t}+\delta\left(R_{x x}+R_{x}^{2}+R_{y y}+R_{y}^{2}\right)-S_{x}^{2}-S_{y}^{2}+\gamma e^{2 R}+\frac{1}{2} \Pi=0, \\
& \quad \Pi_{x x}-\Pi_{y y}+4 \gamma\left(e^{2 R}\right)_{x x}=0 .
\end{aligned}
$$

Setting

$$
\rho=e^{2 R}, \mathbf{v}=-2 \nabla S
$$

leads to a Madelung-type hydrodynamischen system

$$
\begin{aligned}
& \rho_{t}+\nabla(\rho \mathbf{v})=0, \\
& \mathbf{v}_{t}+\mathbf{v} \cdot \nabla \mathbf{v}-\nabla\left[2 \gamma \rho+\Pi+\frac{2 \delta \nabla^{2} \sqrt{\rho}}{\sqrt{\rho}}\right]=\mathbf{0},
\end{aligned}
$$

where

$$
\Pi_{x x}-\Pi_{y y}+4 \gamma \rho_{x x}=0 .
$$

This system was originally introduced in a capillarity model context in [7].

In the $1+1$-dimensional reduction with $\Pi_{y}=0$, the system (8) reduces to the resonant NLS equation. Therein, if $\delta=1-s$ and $s<1$ then reduction may be obtained to the conventional NLS equation. However, if $s>1$ this reduction is not available but, rather, a novel coupled nonlinear system may be derived which admits resonant solitonic behavior. Here, these considerations are extended to the $2+1$-dimensional resonant Davey-Stewartson system (8).

Thus, in the supercritical case $\delta=1-s<0$, on introduction of the scaling

$$
\widetilde{t}=(s-1)^{1 / 2} t, \quad \widetilde{S}=(s-1)^{-1 / 2} S, \quad \widetilde{R}=R,
$$

and setting

$$
e^{+}=e^{R+S}, e^{-}=e^{R-S}
$$

the system (9) may be re-written, on dropping the tilde, as

$$
\begin{aligned}
& -e_{t}^{+}+e_{x x}^{+}+e_{y y}^{+}+\frac{\gamma}{s-1} e^{+} e^{-} e^{+}-\frac{\Pi}{2(s-1)} e^{+}=0, \\
& e_{t}^{-}+e_{x x}^{-}+e_{y y}^{-}+\frac{\gamma}{s-1} e^{-} e^{+} e^{-}-\frac{\Pi}{2(s-1)} e^{-}=0, \\
& \Pi_{x x}-\Pi_{y y}-4 \gamma\left(e^{+} e^{-}\right)_{x x}=0 .
\end{aligned}
$$

The latter system admits a bilinear representation. Thus, if we set

$$
e^{+}=\frac{G^{+}}{F}, e^{-}=\frac{G^{-}}{F}
$$


then $(15)_{1,2}$ produce the bilinear system

$$
\begin{aligned}
& \left(-D_{t}+D_{x}^{2}+D_{y}^{2}\right)\left(G^{+} \cdot F\right)=0, \\
& \left(D_{t}+D_{x}^{2}+D_{y}^{2}\right)\left(G^{-} \cdot F\right)=0, \\
& \left(D_{x}^{2}+D_{y}^{2}+\frac{\Pi}{2(s-1)}\right)(F \cdot F)=\frac{\gamma}{s-1} G^{+} G^{-} .
\end{aligned}
$$

Accordingly, if we set

$$
\Pi=-8(s-1)(\ln F)_{x x}
$$

then the bilinear representation

$$
\begin{gathered}
\left(-D_{t}+D_{x}^{2}+D_{y}^{2}\right)\left(G^{+} \cdot F\right)=0, \\
\left(D_{t}+D_{x}^{2}+D_{y}^{2}\right)\left(G^{-} \cdot F\right)=0, \\
\left(-D_{x}^{2}+D_{y}^{2}\right)(F \cdot F)=\frac{\gamma}{s-1} G^{+} \cdot G^{-}
\end{gathered}
$$

results.

In the subcritical case $s<1$, the transformation

$$
\widetilde{t}=(1-s)^{1 / 2} t, \quad \widetilde{S}=(1-s)^{-1 / 2} S, \quad \widetilde{R}=R
$$

leads to the standard Davey-Stewartson I system with origin in the work of Benney and Roskes [17].

To construct a symmetric 2+1-dimensional version of the Whitham-BroerKaup system, it proves convenient to introduce the variables

$$
\rho=-e^{+} e^{-}, \quad \theta=\frac{-e_{x}^{-}}{e^{-}}, \quad \psi=\frac{-e_{y}^{-}}{e^{-}}
$$

Multiplication of $(15)_{1}$ and $(15)_{2}$ by $e^{-}$and $e^{+}$, respectively, and subtraction yield

$$
\begin{aligned}
\left(e^{+} e^{-}\right)_{t} & =\left(e_{x}^{+} e^{-}-e^{+} e_{x}^{-}\right)_{x}+\left(e_{y}^{+} e^{-}-e^{+} e_{y}^{-}\right)_{y} \\
& =\left[\left(e^{+} e^{-}\right)_{x}+2\left(e^{+} e^{-}\right) \theta\right]_{x}+\left[\left(e^{+} e^{-}\right)_{y}+2\left(e^{+} e^{-}\right) \psi\right]_{y}
\end{aligned}
$$

whence

$$
\rho_{t}=\left(\rho_{x}+2 \rho \theta\right)_{x}+\left(\rho_{y}+2 \rho \psi\right)_{y} .
$$


Moreover,

$$
\begin{aligned}
\theta_{t} & =-\left(\frac{e_{t}^{-}}{e^{-}}\right)_{x}=\left[\left[e_{x x}^{-}+e_{y y}^{-}+\frac{\gamma}{s-1} e^{-} e^{+} e^{-}-\frac{\Pi}{2(s-1)} e^{-}\right] / e^{-}\right]_{x} \\
& =\left(\frac{e_{x x}^{-}}{e^{-}}\right)_{x}+\left(\frac{e_{y y}^{-}}{e^{-}}\right)_{x}+\frac{\gamma}{s-1}\left(e^{+} e^{-}\right)_{x}-\frac{\Pi_{x}}{2(s-1)} \\
& =\left(-\theta_{x}+\theta^{2}\right)_{x}+\left(-\psi_{y}+\psi^{2}\right)_{x}-\frac{\gamma}{s-1} \rho_{x}-\frac{\Pi_{x}}{2(s-1)}
\end{aligned}
$$

so that

$$
\theta_{t}=\left[-\theta_{x}+\theta^{2}-\psi_{y}+\psi^{2}-\frac{\gamma}{s-1} \rho-\frac{\Pi}{2(s-1)}\right]_{x}
$$

where

$$
\theta_{y}=\psi_{x}
$$

and

$$
\Pi_{x x}-\Pi_{y y}+4 \gamma \rho_{x x}=0
$$

In the case of y-independence, (22)-(25) reduce to the Whitham-Broer-Kaup system. Accordingly, the latter and the resonant NLS equation are seen to be equivalent. In 2+1-dimensions, the system (22)-(25) is equivalent to the integrable resonant 2+1-dimensional Davey-Stewartson system introduced by Rogers et al. in [7].

\subsection{Summary}

The system

$$
\begin{aligned}
& \rho_{t}=\left(\rho_{x}+2 \rho \theta\right)_{x}+\left(\rho_{y}+2 \rho \psi\right)_{y}, \\
& \theta_{t}=\left(-\theta_{x}+\theta^{2}-\psi_{y}+\psi^{2}-\frac{\gamma \rho}{s-1}-\frac{\Pi}{2(s-1)}\right)_{x}, \\
& \quad \theta_{y}=\psi_{x}, \\
& \quad-\Pi_{y y}+4 \gamma \rho_{x x}=0
\end{aligned}
$$

constitute a symmetric integrable 2+1-dimensional version of the Whitham-Broer-Kaup system. 
On introduction of a potential $\phi$ according to $\theta=\phi_{x}, \psi=\phi_{y}$ we obtain an alternative Madelung type form of the system (26), namely

$$
\begin{aligned}
\rho_{t}= & \nabla^{2} \rho+2 \nabla \cdot(\rho \nabla \phi), \\
& \phi_{t}-|\nabla \phi|^{2}+\nabla^{2} \phi+\frac{\gamma}{s-1} \rho+\frac{\Pi}{2(s-1)}=0 \\
& \square \Pi+4 \gamma \rho_{x x}=0
\end{aligned}
$$

where

$$
\nabla^{2}:=\partial^{2} / \partial x^{2}+\partial^{2} / \partial y^{2}, \square:=\partial^{2} / \partial x^{2}-\partial^{2} / \partial y^{2}
$$

It is noted that either $\Pi$ or $\rho$ may be eliminated in this system. If $e^{-}$and $e^{+}$ are interchanged in the above procedure then the same system results but with $t \rightarrow-t$.

\section{Solitonic resonance in the 2+1-dimensional Whitham-Broer-Kaup system}

The bilinear representation (19) may now be exploited to construct soliton solutions of the 2+1-dimensional Whitham-Broer-Kaup system (27) via the relations

$$
\begin{gathered}
\phi=-\ln e^{-}=-\ln \left(-G^{-} / F\right), \\
\rho=-\left(e^{+} e^{-}\right)=\frac{-G^{+} G^{-}}{F^{2}}=\frac{1}{F^{2}}\left(D_{x}^{2}-D_{y}^{2}\right)(F \cdot F)\left(\frac{s-1}{\gamma}\right), \\
\Pi=-8(s-1)(\ln F)_{x x} .
\end{gathered}
$$

\subsection{One soliton solution}

The one soliton solution is given by

$$
G^{+}=e^{\eta_{1}^{+}}, \quad G^{-}=-e^{\eta_{1}^{-}}, \quad F=1+\frac{\gamma e^{\eta_{1}^{+}+\eta_{1}^{-}}}{2(s-1)\left[\left(k_{1}^{+}+k_{1}^{-}\right)^{2}-\left(m_{1}^{+}+m_{1}^{-}\right)^{2}\right]}
$$

where $\eta_{1}^{ \pm}=k_{1}^{ \pm} x+m_{1}^{ \pm} y \pm\left(k_{1}^{ \pm 2}+m_{1}^{ \pm 2}\right) t+\eta_{1}^{ \pm(0)}$. Defining

$$
e^{\phi_{11}}=\frac{\gamma}{2(s-1)\left[\left(k_{1}^{+}+k_{1}^{-}\right)^{2}-\left(m_{1}^{+}+m_{1}^{-}\right)^{2}\right]}
$$


we have

$$
\begin{gathered}
\rho=\frac{s-1}{2 \gamma} \frac{\left[\left(k_{1}^{+}+k_{1}^{-}\right)^{2}-\left(m_{1}^{+}+m_{1}^{-}\right)^{2}\right]}{\cosh ^{2} \frac{\eta_{1}^{+}+\eta_{1}^{-}+\phi_{11}}{2}} \\
\Pi=-2(s-1) \frac{\left(k_{1}^{+}+k_{1}^{-}\right)^{2}}{\cosh ^{2} \frac{\eta_{1}^{+}+\eta_{1}^{-}+\phi_{11}}{2}} \\
\phi=-\eta_{1}^{-}+\ln \left(1+e^{\eta_{1}^{+}+\eta_{1}^{-}+\phi_{11}}\right) .
\end{gathered}
$$

Regularity of this solution requires that the parameters be restricted by the inequality $\left|k_{1}^{+}+k_{1}^{-}\right|>\left|m_{1}^{+}+m_{1}^{-}\right|$.

The velocity potential (35) implies that the velocity components determining the form of the domain wall are given by

$$
\begin{gathered}
\theta=\phi_{x}=\frac{k_{1}^{+}-k_{1}^{-}}{2}+\frac{k_{1}^{+}+k_{1}^{-}}{2} \tanh \frac{\eta_{1}^{+}+\eta_{1}^{-}+\phi_{11}}{2} \\
\psi=\phi_{y}=\frac{m_{1}^{+}-m_{1}^{-}}{2}+\frac{m_{1}^{+}+m_{1}^{-}}{2} \tanh \frac{\eta_{1}^{+}+\eta_{1}^{-}+\phi_{11}}{2}
\end{gathered}
$$

(see Figure 1). Introducing amplitudes $k=\frac{k_{1}^{+}+k_{1}^{-}}{2}, m=\frac{m_{1}^{+}+m_{1}^{-}}{2}$, and velocity components $v_{x}=k_{1}^{-}-k_{1}^{+}, v_{y}=m_{1}^{-}-m_{1}^{+}$we have

$$
\theta=-\frac{v_{x}}{2}+k \tanh \left[k\left(x-x_{0}-v_{x} t\right)+m\left(y-y_{0}-v_{y} t\right)\right]
$$

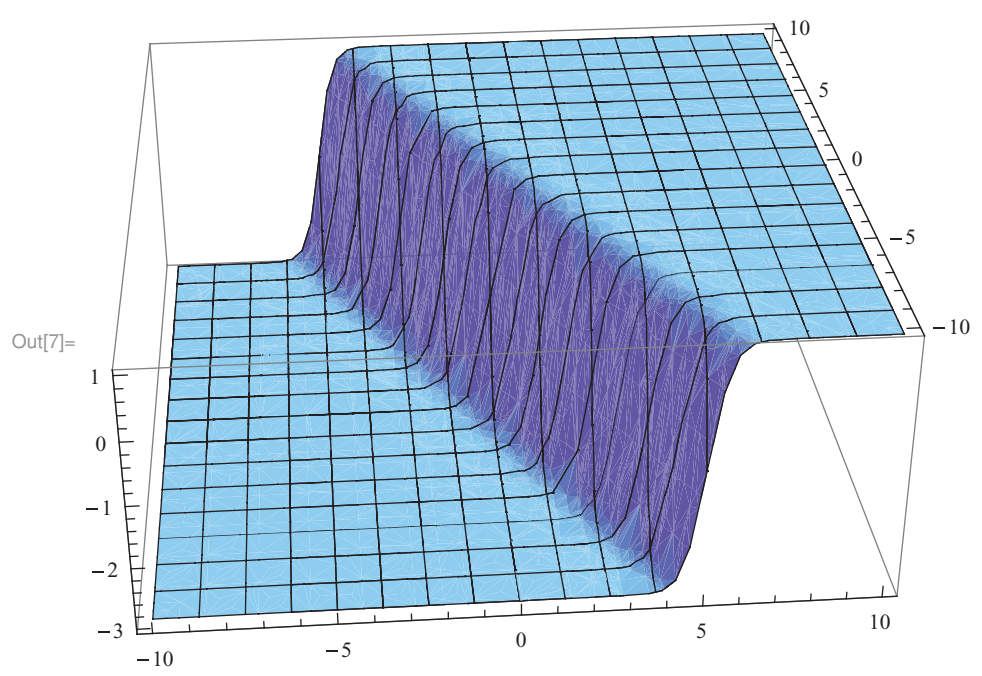

Figure 1. The domain wall for local velocity. $k=2, m=1, v_{x}=2, v_{y}=2$. 


$$
\psi=-\frac{v_{y}}{2}+m \tanh \left[k\left(x-x_{0}-v_{x} t\right)+m\left(y-y_{0}-v_{y} t\right)\right] .
$$

In this solution, the amplitude and the speed of kinks are independent. However, from the regularity condition $|k|>|m|$ mentioned above, it is seen that the velocity field is bounded for $-\frac{v_{x}}{2}-k \leq \theta \leq-\frac{v_{x}}{2}+k,-\frac{v_{y}}{2}-m \leq \psi \leq-\frac{v_{y}}{2}+m$. The domain wall is located along the line $k\left(x-x_{0}-v_{x} t\right)+m\left(y-y_{0}-v_{y} t\right)=0$ and propagates in the direction of the vector $(k, m)$ with constant speed $\left(v_{x}\right.$, $\left.v_{y}\right)$. The density of this soliton solution is shown in Figure 2.

\subsection{Two-soliton resonance}

The two "dissipaton" solution is given by the specializations

$$
\begin{aligned}
& G^{+}=e^{\eta_{1}^{+}}+e^{\eta_{2}^{+}}+\alpha_{1}^{+} e^{\eta_{1}^{+}+\eta_{1}^{-}+\eta_{2}^{+}}+\alpha_{2}^{+} e^{\eta_{2}^{+}+\eta_{2}^{-}+\eta_{1}^{+}} \\
& G^{-}=-\left(e^{\eta_{1}^{-}}+e^{\eta_{2}^{-}}+\alpha_{1}^{-} e^{\eta_{1}^{+}+\eta_{1}^{-}+\eta_{2}^{-}}+\alpha_{2}^{-} e^{\eta_{2}^{+}+\eta_{2}^{-}+\eta_{1}^{-}}\right) \\
& F=1+\beta_{1} e^{\eta_{1}^{+}+\eta_{1}^{-}}+\beta_{2} e^{\eta_{2}^{+}+\eta_{1}^{-}}+\beta_{3} e^{\eta_{1}^{+}+\eta_{2}^{-}}+\beta_{4} e^{\eta_{2}^{+}+\eta_{2}^{-}}+\beta_{5} e^{\eta_{1}^{+}+\eta_{1}^{-}+\eta_{2}^{+}+\eta_{2}^{-}},
\end{aligned}
$$

where $\eta_{i}^{ \pm}=k_{i}^{ \pm} x+m_{i}^{ \pm} y \pm\left(k_{i}^{ \pm 2}+m_{i}^{ \pm 2}\right) t+\eta_{i}^{ \pm(0)}, i=1,2$ together with

$$
\begin{gathered}
\alpha_{1}^{+}=\frac{\gamma}{2} \frac{\left(\breve{k}_{12}^{++}\right)^{2}-\left(\check{m}_{12}^{++}\right)^{2}}{\left(\left(k_{11}^{+-}\right)^{2}-\left(m_{11}^{+-}\right)^{2}\right)\left(\left(k_{21}^{+-}\right)^{2}-\left(m_{21}^{+-}\right)^{2}\right)} \\
\alpha_{2}^{+}=\frac{\gamma}{2} \frac{\left(\check{k}_{12}^{++}\right)^{2}-\left(\check{m}_{12}^{++}\right)^{2}}{\left(\left(k_{12}^{+-}\right)^{2}-\left(m_{12}^{+-}\right)^{2}\right)\left(\left(k_{22}^{+-}\right)^{2}-\left(m_{22}^{+-}\right)^{2}\right)} \\
\alpha_{1}^{-}=\frac{\gamma}{2} \frac{\left(\breve{k}_{12}^{--}\right)^{2}-\left(\check{m}_{12}^{--}\right)^{2}}{\left(\left(k_{11}^{+-}\right)^{2}-\left(m_{11}^{+-}\right)^{2}\right)\left(\left(k_{12}^{+-}\right)^{2}-\left(m_{12}^{+-}\right)^{2}\right)} \\
\alpha_{2}^{-}=\frac{\gamma}{2} \frac{\left(\check{k}_{12}^{--}\right)^{2}-\left(\check{m}_{12}^{--}\right)^{2}}{\left(\left(k_{21}^{+-}\right)^{2}-\left(m_{21}^{+-}\right)^{2}\right)\left(\left(k_{22}^{+-}\right)^{2}-\left(m_{22}^{+-}\right)^{2}\right)} \\
\beta_{1}=\frac{\gamma}{2\left[\left(k_{11}^{+-}\right)^{2}-\left(m_{11}^{+-}\right)^{2}\right]} \\
\beta_{2}=\frac{\gamma}{2\left[\left(k_{21}^{+-}\right)^{2}-\left(m_{21}^{+-}\right)^{2}\right]} \\
\beta_{3}=\frac{\gamma}{2\left[\left(k_{12}^{+-}\right)^{2}-\left(m_{12}^{+-}\right)^{2}\right]} \\
\beta_{4}=\frac{\gamma}{2\left[\left(k_{22}^{+-}\right)^{2}-\left(m_{22}^{+-}\right)^{2}\right]}
\end{gathered}
$$




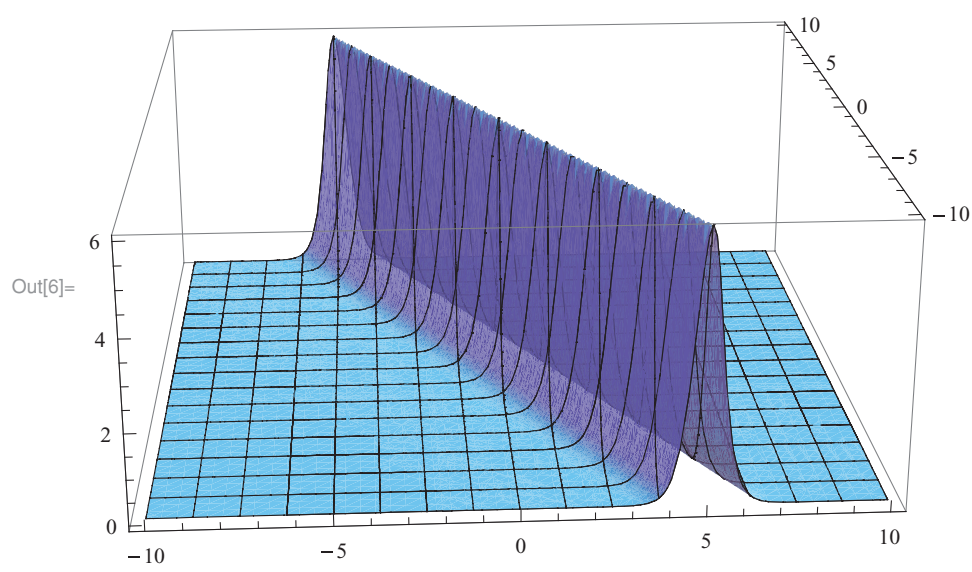

Figure 2. The density as a line soliton. $k=2, m=1, s=2, \gamma=1, v_{x}=2, v_{y}=2$.

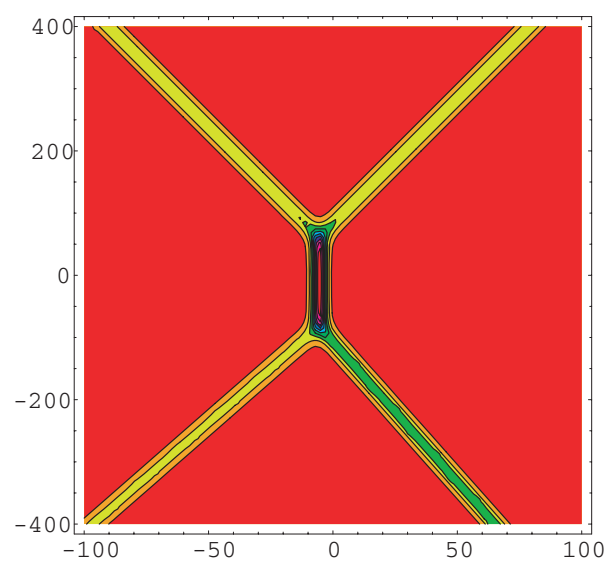

Figure 3. The two-soliton resonance.

$\beta_{5}=\frac{\gamma^{2}\left[\left(\check{k}_{12}^{++}\right)^{2}-\left(\check{m}_{12}^{++}\right)^{2}\right]\left[\left(\check{k}_{12}^{--}\right)^{2}-\left(\check{m}_{12}^{--}\right)^{2}\right]}{4\left[\left(k_{11}^{+-}\right)^{2}-\left(m_{11}^{+-}\right)^{2}\right]\left[\left(k_{21}^{+-}\right)^{2}-\left(m_{21}^{+-}\right)^{2}\right]\left[\left(k_{12}^{+-}\right)^{2}-\left(m_{12}^{+-}\right)^{2}\right]\left[\left(k_{22}^{+-}\right)^{2}-\left(m_{22}^{+-}\right)^{2}\right]}$

In this solution, for certain values of the parameters, $\beta_{5}$ can vanish or become infinite. In such cases the two-soliton solution fuses to create a single soliton solution corresponding to "resonant" soliton interaction.

In Figure 3, the density in a case of two-soliton resonance is shown in the absence of loops, at values of parameters $k_{1}^{+}=0.9, k_{1}^{-}=-0.5, k_{2}^{+}=0.1$, $k_{2}^{-}=-0.45, m_{1}^{+}=0.2, m_{1}^{-}=-0.1, m_{2}^{+}=0.2, m_{2}^{-}=-0.1, \eta_{1}^{+(0)}=-7$, $\eta_{2}^{+(0)}=4, \eta_{1}^{-(0)}=-7, \eta_{2}^{-(0)}=4$. 


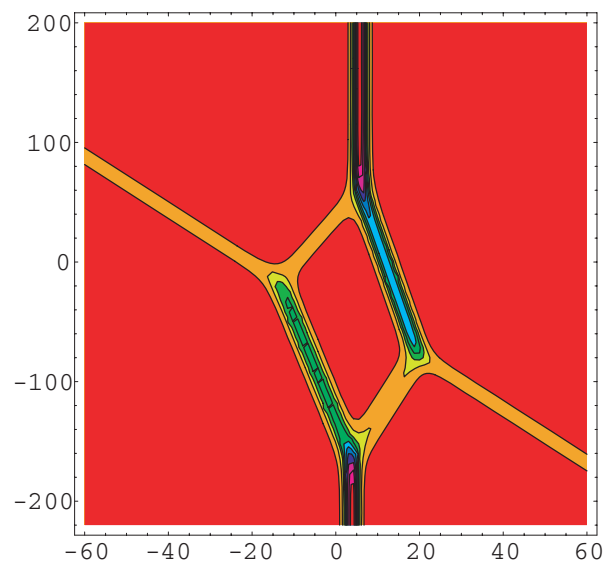

Figure 4. The four-soliton resonance.

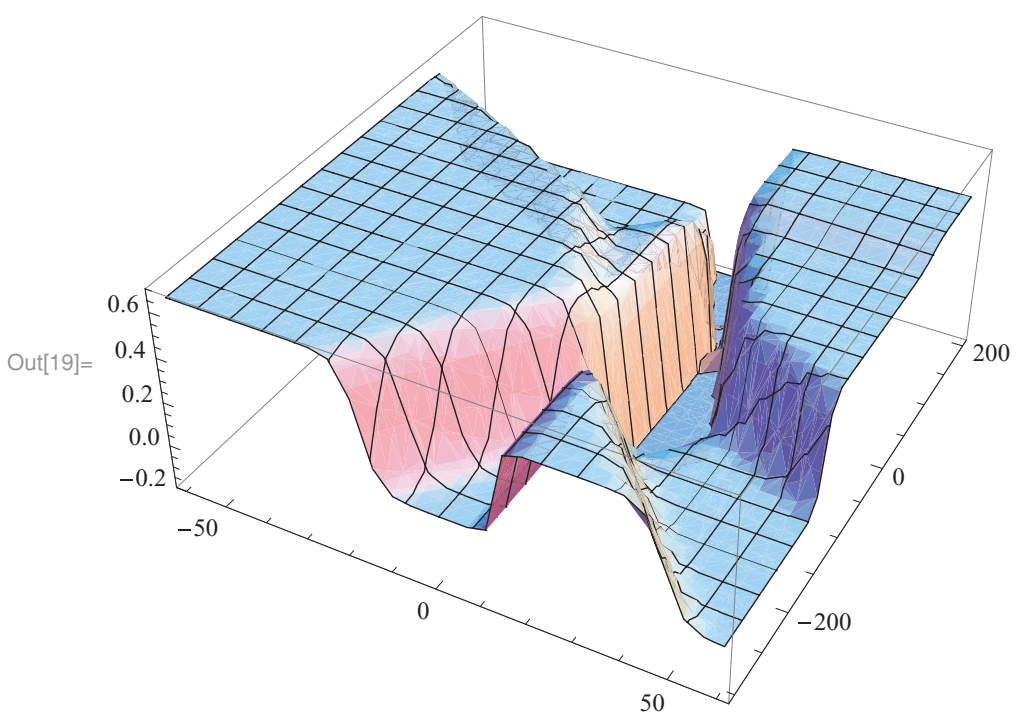

Figure 5. The four-soliton resonance speed.

In Figure 4, the density is plotted for four-soliton resonance with one loop corresponding to values of parameters $k_{1}^{+}=0.5, k_{1}^{-}=0.9, k_{2}^{+}=0.1$, $k_{2}^{-}=0.35, \quad m_{1}^{+}=0.1, \quad m_{1}^{-}=-0.1, \quad m_{2}^{+}=0.2, \quad m_{2}^{-}=10^{-7}, \quad \eta_{1}^{+(0)}=10$, $\eta_{2}^{+(0)}=10, \eta_{1}^{-(0)}=0, \eta_{2}^{-(0)}=0$.

In Figure 5, the velocity field component in the $\mathrm{x}$ direction is plotted for four-soliton resonance at values of parameters $k_{1}^{+}=0.5, k_{1}^{-}=0.9$, $k_{2}^{+}=0.1, k_{2}^{-}=0.35, m_{1}^{+}=0.1, m_{1}^{-}=-0.1, m_{2}^{+}=0.2, m_{2}^{-}=0.001, \eta_{1}^{+(0)}=$ $0, \eta_{2}^{+(0)}=0, \eta_{1}^{-(0)}=0, \eta_{2}^{-(0)}=0$ 


\title{
Acknowledgment
}

One of the authors (C.R.) acknowledges with gratitude support under Hong Kong Research Grant Council Project No: 502009.

\section{References}

1. D. J. KaUp, A higher-order water wave equation and the method for solving it, Prog. Theor. Phys. 54:396-408 (1975).

2. D. J. KAUP, Finding eigenvalue problems for solving nonlinear evolution equations, Prog. Theor. Phys. 54:72-78 (1975).

3. L. J. F. BROER, Approximate equations for long water waves, Appl. Sci. Res. 31:377-395 (1975).

4. L. J. F. Broer, E. W. C. Van Groessen, and J. M. W. Timmers, Stable model equations for long water waves, Appl. Sci. Res. 32:618-636 (1976).

5. R. A. Leo, G. Mancarella, and G. Soliani, On the Broer-Kaup hydrodynamical system, J. Phys. Soc. Japan 57:753-756 (1988).

6. G. B. Whitham, Variational methods and applications to water waves, Proc. Roy. Soc. A 299:6-25 (1967).

7. C. Rogers, L. P. YIP, and K. W. ChOw, A resonant Davey-Stewartson capillarity model system. Soliton generation, Int. J. Nonlinear Sci. Num. Simulation 10:397-405 (2009).

8. Z. F. Liang and X. Y. TANG, Painlevé analysis and exact solutions of the resonant Davey-Stewartson system, Phys. Lett. A 274:110-115 (2009).

9. L. De Broglie, La mécanique ondulatoire et la structure atomique de la matiére et du rayonnement, Journal de Physique et du Radium 8:225-241 (1927).

10. D. BoHm, A suggested interpretation of the quantum theory in terms of hidden variables, I and II, Phys. Rev. 85:166-193 (1952).

11. W. G. Wagner, H. A. Haus, and J. H. Marburger, Large-scale self-trapping of optical beams in the paraxial ray approximation, Phys. Rev. 175:256-266 (1968).

12. C. Rogers, B. Malomed, K. Chow, and H. An, Ermakov-Ray-Reid systems in nonlinear optics, J. Phys. A: Math. Theor. 43:455214 (2010).

13. O. K. Pashaev and J. H. LeE, Resonance solitons as black holes in Madelung fluid, Mod. Phys. Lett A 17:1601-1619 (2002).

14. J. H. Lee, O. K. Pashaev, C. Rogers, and W. K. Schief, The resonant nonlinear Schrödinger equation in cold plasma physics. Application of Bäcklund transformations and superposition principles, J. Plasma Phys. 73:257-272 (2007).

15. C. Rogers and W. K. SCHIEF, The resonant nonlinear Schrödinger equation via an integrable capillarity model, Il. Nuovo Cimento 114B:1409-1412 (1999).

16. O. K. Pashaev, J. H. Lee, and C. Rogers, Soliton resonances in a generalised nonlinear Schrödinger equation, J. Phys. A. Math. Theor. 41:452001-452009 (2008).

17. D. J. BenNey and G. J. Roskes, Wave instabilities, Stud. Appl. Math. 48:377-385 (1969).

\author{
The Hong Kong Polytechnic University \\ and The University of New South Wales \\ IZMir Institute of TeChNOLOGY
}

(Received November 17, 2010) 